\section{Scanning beyond the limits of standard OCT with a Fourier domain optical coherence tomography integrated into a slit lamp: the SL SCAN-1}

\begin{abstract}
Purpose With a new Fourier domain optical coherence tomography (FD OCT) device, SL

SCAN-1 (Topcon Europe Medical BV, Capelle a/d IJssel, The Netherlands), integrated into a slit-lamp OCT, scans can be obtained through a handheld lens. The necessary adjustment of the reference arm is possible by fast Z-alignment. This study was performed to prove the capability of SL SCAN-1 to scan through a three-mirror contact lens, scanning the peripheral retina and anterior chamber angle.

Methods Different representative pathologies of the peripheral retina and anterior chamber were simultaneously observed and scanned with the SL SCAN-1. The scans of peripheral retinal lesions were obtained both through a handheld lens and through a three-mirror contact lens. The anterior chamber angle was scanned directly with the SL SCAN-1 in anterior mode, and through the gonio-mirror of a three-mirror contact lens with the SL SCAN-1 in posterior mode.
\end{abstract}

Results OCT scans could be obtained with the SL SCAN-1 of the peripheral retina through both, a common handheld lens and a three-mirror contact lens. The scans obtained through a three-mirror contact lens were of better quality, visualizing details of the different layers of the retina more clearly. The scans of the anterior chamber, obtained through the gonio-mirror of a three-mirror contact lens, visualized the open anterior chamber angle, with details of fine structures.

M Stehouwer ${ }^{1}$, FD Verbraak ${ }^{1,2}$, HR de Vries $^{3}$ and TG van Leeuwen ${ }^{2}$

Conclusions The SL SCAN-1 is a unique FD OCT system, which is able to scan not only the posterior pole and anterior segment but also the anterior chamber angle and the more peripheral retina. These four modalities combined into one device could make the SL SCAN-1 a very powerful aid in daily practice. Eye (2011) 25, 97-104; doi:10.1038/eye.2010.162; published online 12 November 2010

Keywords: Fourier domain optical coherence tomography; slit lamp; SL SCAN-1; three-mirror contact lens; posterior segment; anterior segment

\section{Introduction}

During the last decade, optical coherence tomography (OCT) has become an almost indispensable imaging technique in ophthalmology. By obtaining highly detailed cross-sectional images of the examined tissue, it is an important investigation tool not only for diagnosis and follow-up of retinal diseases but also for glaucoma and diseases of the ocular anterior segment. Recently, we described a Fourier domain (FD) OCT integrated into a common slit lamp (Topcon SL-D7), the SL SCAN-1 (Topcon). ${ }^{1}$ With the slit-lampintegrated OCT, one can obtain OCT images of the posterior or anterior segment during a normal standard examination of the eye. This increases the efficiency of the routine clinical examination of a patient, will increase the
'Department of Ophthalmology, Academic Medical Center, University of Amsterdam, Amsterdam, The Netherlands

${ }^{2}$ Department of Biomedical Engineering and Physics, Academic Medical Center, University of Amsterdam, Amsterdam

The Netherlands

${ }^{3}$ Topcon Europe Medical BV, Capelle a/d IJssel,

The Netherlands

Correspondence: M Stehouwer, Department of Ophthalmology, Academic Medical Center, University of Amsterdam, Meibergdreef 9, Amsterdam 1105 AZ, The Netherlands Tel: + 31-20-5663751;

Fax: +31-20-5669053.

E-mail:m.stehouwer@ amc.uva.nl

Received: 12 April 2010 Accepted in revised form: 31 August 2010 Published online: 12 November 2010 


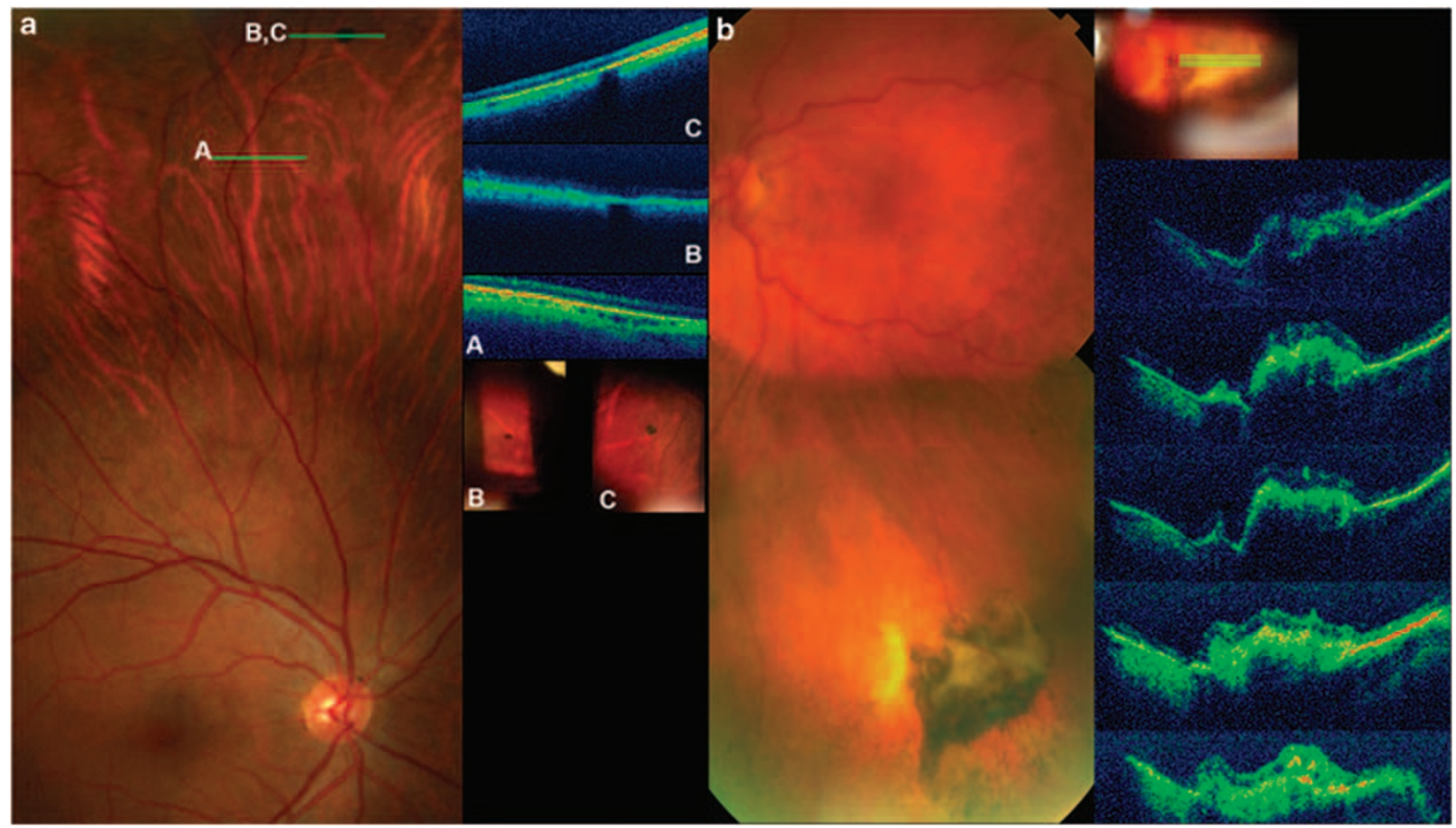

Figure 1 Scanning outside the standard range of the common commercially available (posterior segment) OCT devices. (a) Peripheral naevus; scan A: the most peripheral scan that could be obtained with the 3D-OCT-1000 Mark II, Topcon; scan B: scan obtained with SL SCAN-1 through a Volk Superfield lens; scan C: scan obtained with SL SCAN-1 through a three-mirror contact lens (HAAG-STREIT). (b) Peripheral lesion suspect for a metastasis; scans obtained through a Volk Superfield lens.

comfort of the patient seated behind just one device, and will also save time.

The SL SCAN-1 takes an OCT scan in an area of interest at exactly the position of an aiming line observed through the right ocular during slit lamp biomicroscopy. The focus of the OCT in posterior mode is adjusted with the aid of a fast Z-alignment in the reference arm of the device. Through this Z-alignment, one can obtain OCT scans of the posterior segment with any common handheld lens, while simultaneously performing slit-lamp biomicroscopy of the fundus. In the anterior mode, the fast Z-tracking system is switched off. The focus of the slit lamp and the OCT is then fixed and coincided, and OCT scans of the anterior segment observed with the slit lamp is obtained directly.

While evaluating the limits of clinical performance of the SL SCAN-1, it was soon recognized that the integration of the OCT into a slit lamp expands the range of the scanned area of the retina compared with the range of the common commercially available OCT devices (Figure 1a, scan A and B). Not only the posterior pole but also the periphery comes within reach. Basically, the rule is: 'what you can see is what you can scan'.

Till now cross-sectional images of the peripheral retina could only be obtained with ultrasonographic biomicroscopy (UBM) or MRI, with a rather low resolution. The SL SCAN-1 can provide cross-sectional images with much higher resolution, showing details of the different layers of the peripheral retina. This could provide new insights and additional information of peripheral retinal abnormalities and diseases.

Although OCT scans of peripheral lesions in the retina can be obtained through a handheld lens, the quality of the scans seemed to be inversely related to the distance from the fovea. Most likely, this inverse relationship is the result of an increase in optical aberrations, introduced by the lens and optics of the examined eye, when observing the more peripheral retina.

An improvement in the optics, observing the peripheral retina, could be accomplished by using a three-mirror contact lens. Because the SL SCAN-1 is capable of scanning through a handheld lens by the fast Z-alignment of the reference arm, it was hypothesized that the SL SCAN-1 is capable of scanning the more peripheral retina through a three-mirror contact lens. In addition, the anterior chamber angle can be observed with the gonio-mirror of the three-mirror contact lens, and, probably, OCT scans could be taken of the structures in the anterior chamber angle such as the trabecular meshwork.

The aim of this paper is to describe the first impressions of OCT scans performed through a 
three-mirror contact lens using the SL SCAN-1, enabling detailed images of peripheral retinal lesions and images of the anterior chamber angle.

\section{Subjects and methods}

\section{Subjects}

Patients with various representative pathologies of the anterior chamber angle and periphery of the retina seen in the outpatient clinic of the ophthalmic department of the Academic Medical Center, Amsterdam the Netherlands, between June and October 2009, were asked to participate in an ongoing study to evaluate the SL SCAN-1. Participating patients were diagnosed with pigment dispersion syndrome $(n=2)$; primary open angle glaucoma, status after Baerveldt implant procedure $(n=2)$; peripheral lesions, naevus $(n=1)$, lesion suspect for a metastasis $(n=1)$; peripheral retinal defect, status after laser treatment $(n=2)$; and peripheral schisis $(n=2)$. These patients were selected to provide the first impression of OCT scans obtained through the threemirror contact lens.

The study followed the tenets of the declaration of Helsinki. Patients were informed about the nature of the study and gave their written informed consent. Patients with pathology in the peripheral retina received mydriatic eyedrops in the affected eye (tropicamide and phenylephrine). All patients received two drops of oxybuprocaïne to anaesthetize the eye before applying the three-mirror contact lens with methocel $2 \%$.

\section{SL SCAN-1; imaging through three-mirror contact lens}

All images were acquired using SL SCAN-1 as described in a previous paper. ${ }^{1}$ Briefly, SL SCAN-1 is a Fourier domain optical coherence tomograph (FD OCT) with a broadband superluminescent diode light source $(\Delta \lambda 30 \mathrm{~nm}$, central wavelength $830 \mathrm{~nm})$. The scan resolution is $8-9 \mu \mathrm{m}$ in tissue, with a scan speed of 5000 a-scans per second. Each b-scan is composed of 512 a-scans, scan length is up to $12 \mathrm{~mm}$, and scan depth is $2 \mathrm{~mm}$ in tissue. Positioning of the b-scan is accomplished with the help of an aiming line visible through one of the oculars. The slit lamp and lens provide considerable flexibility to position the aiming line and a fixation target is not necessary. Obtaining OCT scans simultaneously with slit-lamp biomicroscopy will take only a few extra seconds. Photographs can be taken simultaneously of all lesions scanned with a slit-lamp-mounted digital camera, the DV-3 (3.4 mega pixel,Topcon).

Scans of the posterior segment are made through a handheld lens, while the (lesion in the) retina is simultaneously observed with the slit lamp.
Slight movements of the patient, handheld lens, and/or slit lamp during examination result in fluctuations in the length of the sample arm. With the SL SCAN-1 in posterior mode, a fast Z-tracking system in the reference arm is switched on. This system compensates for the dynamic character of the examination and keeps the position of the retina in the live window at the desired position.

The length of the sample arm will differ considerably between a handheld lens and a three-mirror contact lens. However, independent of the used lens and its corresponding sample arm length, the fast Z-tracking system is able to line out the correct length of the reference arm. Once the retinal signal is found, the system will lock on to this signal and will continuously compensate for the dynamic character of the examination. The range of the standard Z-tracking system was slightly enlarged in this study, enabling the SL SCAN-1 to line out the correct length of the reference arm while using a three-mirror contact lens. This range adjustment can be made with any SL SCAN-1 device. To obtain OCT scans through a three-mirror contact lens, with the gonio-mirror and the peripheral retina mirror, the SL SCAN-1 is used in posterior mode (Z-tracking system switched on).

In this study, scans of peripheral retinal lesions were performed both with a handheld lens (Volk SuperField NC Lens) and with a three-mirror contact lens (HAAG-STREIT AG, Koeniz, Switzerland). Scans of the anterior chamber angle were taken directly with the SL SCAN-1 in anterior mode (Z-alignment switched off), and through the gonio-mirror of a three-mirror contact lens with the SL SCAN-1 in posterior mode (Z-alignment switched on). Different slit-lamp magnifications were used during scanning, from $\times 10$ to $\times 40$. The angle of the scan direction was either perpendicular or parallel to the ora serrata while scanning the peripheral retina, or to the anterior chamber angle while scanning the anterior chamber structures.

\section{Results}

The use of the slit lamp (Topcon SL-D7) with the add-on SL SCAN-1 was almost identical to normal routine slit lamp examination, either with a handheld lens or with a three-mirror contact lens. No limitations were observed during any examination. The area of interest could be brought into focus; the aiming line was clearly visible and simultaneous observing and scanning through any of the three mirrors of a three-mirror contact lens (HAAG-STREIT) could be performed.

The SL SCAN-1 is capable of scanning outside the standard range of the common commercially available (posterior segment) OCT devices. Figure 1a shows a scan 


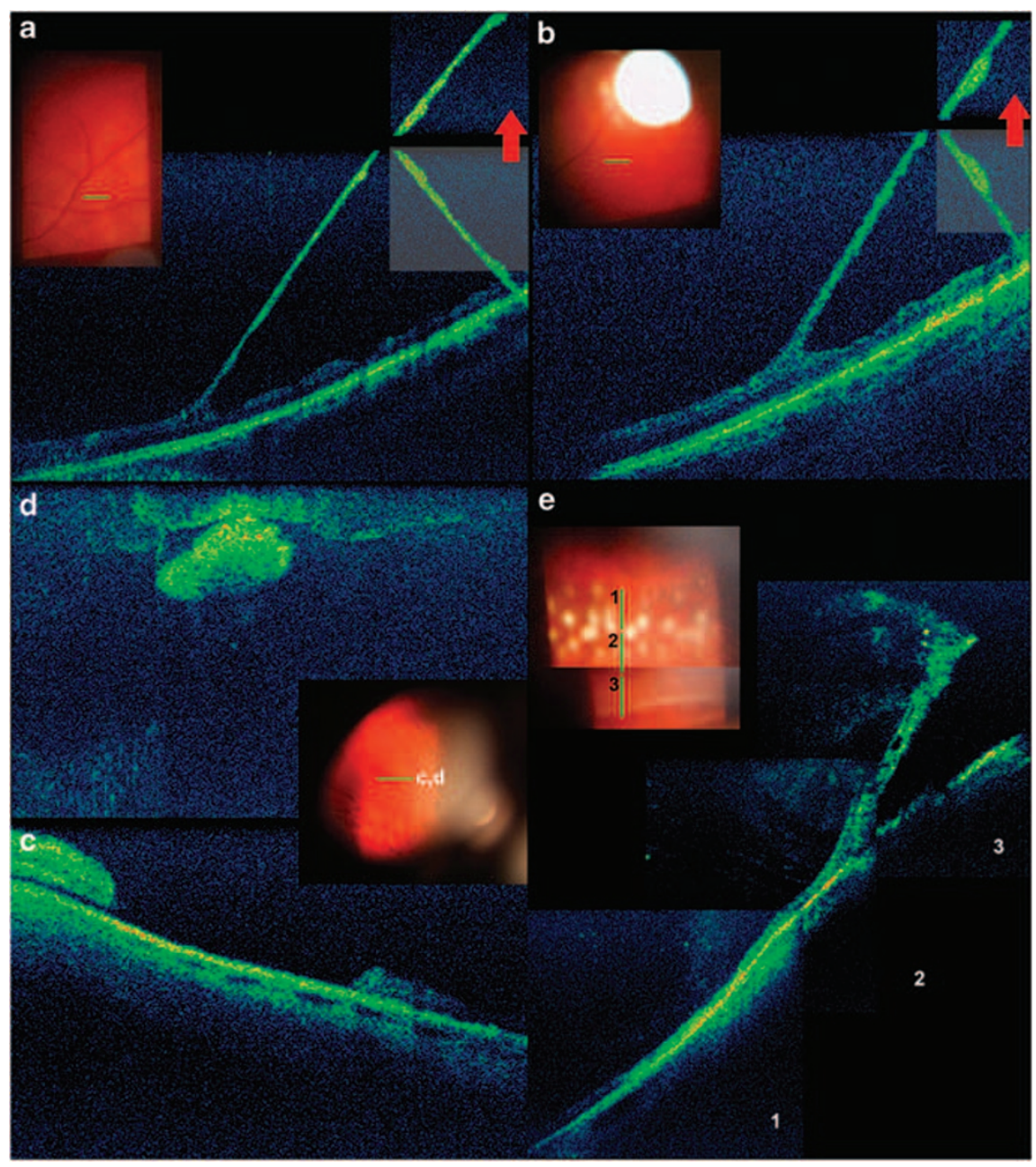

Figure 2 (a) Retinoschisis: scans obtained through a Volk Superfield lens and (b) through a three-mirror contact lens (HAAGSTREIT). Arrow: interpretation of the mirrored structures, in the literature known as 'complex conjugate (mirror) image artefact'. (c and d) Peripheral retinal U-tear surrounded with attached retina (c) and the rumpled flap (d). (e) Peripheral retina detachment; status after laser treatment. Composition of three scans obtained through a three-mirror contact lens (HAAG-STREIT).

obtained through a handheld lens of a retinal lesion (naevus) anterior to the equator (scan B). The expected shadowing below the naevus is visible but the image lacks fine details of the retina. In contrast, the scan performed through a three-mirror contact lens showed not only the characteristic shadowing but also details of the different layers of the retina in this location (Figure 1a, scan C). A patient with a mamacarcinoma and lung metastases was seen in the outpatient clinic for diabetic retinopathy, and for a suspicious lesion in the periphery of the right eye. The OCT showed an elevated lesion without leakage on top (Figure 1b), confirmed on late-phase fluorescein angiography. Final diagnosis revealed a peripheral choroidal neovascularization. Figures $2 a$ and $b$ show the obtained scans of peripheral retinoschisis with a clear splitting of the retina into an inner and outer retinal layer. The obtained scans of a peripheral retinal U-tear treated with laser show the defect surrounded by the attached retina (Figure 2c) and the rumpled flap (Figure 2d). In a patient with a lasered peripheral retinal giant tear, scans were taken from the attached to the detached retina. An image composed of a few scans shows the attached retina with the surrounding ring of irregular retina as a consequence of the laser coagulates and the detached retina with vitreoretinal traction (Figure 2e). Such scans can provide information about the efficacy of the laser coagulate boundary.

Figures $3 a$ and $b$ show images of a patient with $a$ normal anterior chamber angle. The image obtained with the SL SCAN-1 in anterior mode (Figure 3a) shows an open anterior chamber angle, without details of the 


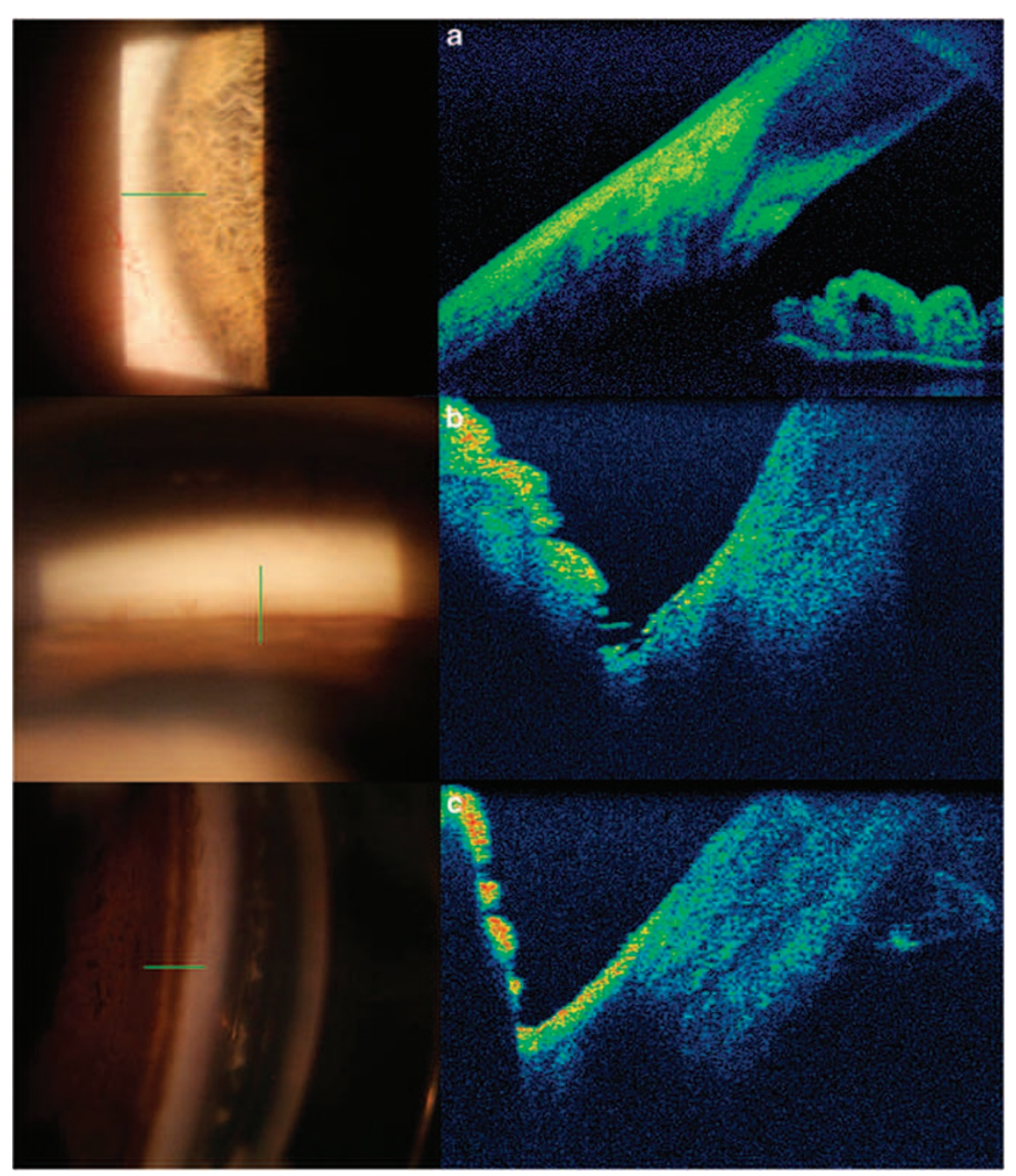

Figure 3 (a) Open anterior chamber angle; scan obtained with SL SCAN-1 in anterior mode. (b) Visualization of iris processes and the triangular trabecular meshwork; scan obtained through the gonio-mirror of the three-mirror contact lens with the SL SCAN-1 in posterior mode. (c) Higher reflectivity of the triangular trabecular meshwork because of the accumulation of pigment in a patient with pigment dispersion.

structures composing the angle itself. The complete structure of the angle became visible in the images obtained through the gonio-mirror of the three-mirror contact lens, with the SL SCAN-1 in posterior mode and the slit-lamp magnification at $\times 10$. With the slit-lamp magnification at $\times 40$, iris processes and the triangular trabecular meshwork were visualized (Figure $3 b$ ).

In patients with pigment dispersion, this triangular trabecular structure demonstrated a higher reflectivity because of the accumulation of pigment in the trabecular meshwork, which is typical for this disease (Figure 3c).

\section{Discussion}

This study demonstrated the proof of principle that the SL SCAN-1 can obtain OCT scans of the peripheral retina and the anterior chamber angle through a three-mirror contact lens. The add-on OCT did not interfere with the functionality of the slit lamp during examination of the retina or the anterior chamber through a three-mirror contact lens; OCT scans could be obtained of all structures that were observed during slit-lamp biomicroscopy through the three-mirror contact lens. Basically, the rule is: "what you can see is what you can scan'.

To examine lesions in the peripheral retinal, the ophthalmologist can use fundoscopy, photography (with or without angiography), UBM, or MRI to come to a final diagnosis. None of these examinations provide detailed cross-sectional images of the peripheral retina. The OCT scans of the peripheral retina taken with the SL SCAN-1 do show retinal structures with a resolution of $15-20 \mu \mathrm{m}$. 
These detailed images of the peripheral retina will provide additional information on retinal diseases such as peripheral degenerations, retinoschisis, Coats disease, peripheral retinal angiomatous proliferations, and peripheral neovascularizations. OCT scans of the peripheral retina would also aid in the diagnosis and follow-up of peripheral retinal defects. The presence of traction on a peripheral retinal defect is of importance in treatment choice and frequency of follow-up. It will help in the evaluation of the efficacy of laser treatment, determining whether there is progressive leakage beyond the laser coagulates. In peripheral tumours, the presence of leakage on top of the tumour is of importance in differential diagnostics. As demonstrated in Figure 1, in a patient with peripheral neovascularization, this important information can be obtained with the SL SCAN-1.

Examinations of the anterior chamber angle can be performed with gonioscopy, UBM, adjusted posterior segment OCT devices, and dedicated anterior segment OCT devices. The best resolution is obtained with the dedicated anterior segment OCT devices. However, the anterior chamber angle structures are, for a large part, obscured by the high scattering properties of the peripheral cornea. With the SL SCAN-1, OCT scans of the anterior chamber can be obtained through the goniomirror of a three-mirror contact lens. This could provide images of peripheral anterior synechiae, the structural abnormalities in goniodysgenesis (Rieger's anomaly, Peter's anomaly, congenital glaucoma), and of tumours in the angle, such as extensions of iris or ciliary body melanomas. By scanning through a three-mirror contact lens, bypassing the high scatter properties, the trabecular meshwork structure becomes visible. Figure $3 \mathrm{c}$ shows an increased reflectivity of the triangular trabecular structure due to accumulation of pigment in this meshwork in a patient with pigment dispersion. Visualizing the changes in the trabecular meshwork could yield more information about the pathophysiology in pigment dispersion, pseudo exfoliation, and perhaps even show changes due to corticosteroids in so-called high responders. ${ }^{2,3}$

The SL SCAN-1 is a unique FD OCT system that can scan the posterior pole and anterior segment, and also the anterior chamber angle and the more peripheral retina. These four modalities combined into one device makes the SL SCAN-1 a very powerful aid in daily practice. The possibility to take photographs of all lesions observed and scanned is another important asset of the system. All OCT images and photographs can be taken during the normal standard examinations with the slit lamp. This increases the efficiency of the routine clinical examination of a patient. In many clinics, OCT examinations are performed by technicians who are not directly familiar with the pathology at hand. This could lead to inadequate scans missing the relevant pathology. This problem is avoided with the slit lamp with integrated OCT, when the ophthalmologist himself will perform the OCT scans, directing the scan to the area of interest observed with slit-lamp biomicroscopy. The ophthalmologist can directly interpret the obtained scans, and use the images to explain a diagnosis or a treatment to the patient.

Similar to most of the FD OCT retina-dedicated systems, SL SCAN-1 has a limited imaging depth range of $2 \mathrm{~mm} .{ }^{4-6}$ Sometimes a symmetric overlapping image artefact, referred to in literature as the complex conjugate or mirror image artefact, can show up in OCT images. ${ }^{7,8}$ However, a healthy retina is much thinner than $2 \mathrm{~mm}$ and, by keeping the patient's retina completely on one side of the reference position, this artefact will normally not show up in the scans of the posterior segment. An example of the mirror image artefact is shown in Figures 2a and b, showing a mirroring of the inner layer of the retinoschisis. Because the anterior chamber has a depth up to $6 \mathrm{~mm}$, this mirror image artefact is present in most images of the anterior segment. This partly mirrored image will show up in overview images of the cornea, together with the iris (slit-lamp magnification of $\times 10$, scan length $12 \mathrm{~mm}$ ). The artefact can be avoided in images of the anterior segment by obtaining scans with a higher slit-lamp magnification; by this, the imaged structure can be positioned completely on one side of the reference position (Figure 3a). In this study, the obtained images were not partly mirrored in images taken with the SL SCAN-1 in anterior mode, using a slit-lamp magnification of $\times 25$ or higher and a scan length of $4 \mathrm{~mm}$. Similarly, the obtained scans through a three-mirror contact lens of the anterior chamber angle did not show the symmetric overlapping image artefact with a slit-lamp magnification of $\times 25$ or higher.

Images of the posterior segment with SL SCAN-1, similar to other posterior segment OCT systems, seem to be in accordance with the anatomy of the retina. However, the refraction of light through the sample arm is of importance in the configuration of the obtained image. This influence is more pronounced in images taken with the SL SCAN-1 of the anterior segment. The direction of the b-scan in relation to the scanned structures of the anterior segment through a three-mirror contact lens will add an extra complexity to the interpretation of the b-scans. A scan performed perpendicular to the anterior angle will result in a b-scan with the cornea on one side, the iris at the opposite side, and the angle in the middle (Figure $3 b$ ). Some basic knowledge of the OCT technology and experience in 
practice will be necessary to obtain the desired scans and for a correct interpretation of the images.

In this study, we attempted to obtain comparable peripheral OCT scans with the 3D-OCT-1000 Mark II, Topcon. It was impossible to obtain OCT scans of the peripheral retina, even when the patient was asked to gaze far to the side (Figure 1a, scan A). Other imaging modalities such as UBM and MRI do not have the high resolution of an FD OCT system, and were not an alternative for comparison.

There is rapid development in anterior segment imaging. ${ }^{7,9-11}$ In addition to TD-OCT systems (Visante, Zeiss; Anterior Segment OCT, Heidelberg, Germany), a dedicated anterior segment FD OCT system is now commercially available (Casia SS-1000, Tomey Corporation, Nagoya, Japan). ${ }^{12,13}$ Commercially available posterior segment OCT systems can also be modified to take scans of the anterior segment. ${ }^{14}$ Detailed images of the anterior chamber angle can be taken, even visualizing Schlemm's canal. ${ }^{11,14}$ Images of the anterior chamber angle, taken with SL SCAN-1 through a three-mirror contact lens, did show fine structures such as the trabecular meshwork. Taking into account the fact that this contact method is generally less desirable, it carries no great risks for the patient. Further studies have to be performed to evaluate whether new insights in anterior chamber angle pathology can be obtained by scanning through a gonio-mirror, and whether this technique provides more information than the currently available systems.

In conclusion, SL SCAN-1 is equipped with a fast Z-alignment system in the reference arm, which makes it possible to obtain good-quality OCT scans through a three-mirror contact lens. Scanning through a threemirror contact lens enables to scan the peripheral retina and the anterior chamber angle structures. This FD OCT system has the unique combination of four modalities, namely, the ability to scan the retina posterior pole through a handheld lens, the peripheral retina through a three-mirror contact lens, the anterior segment directly, and the anterior chamber angle through the gonio-mirror of a three-mirror contact lens. High-resolution OCT images of peripheral lesions with enough detail to show the different retinal layers are presented in this paper. Future clinical studies have to prove the usefulness of these scans in the management of patients. The ability to obtain in vivo images of lesions in the peripheral retina with a resolution comparable to histology will lead to new insights into the pathophysiology of these lesions. Similarly, scans of the anterior chamber angle, taken through the three-mirror contact lens, visualizing anterior chamber structures such as iris processes and the trabecular meshwork could provide new and valuable information.

\section{Summary}

\section{What was known before}

- Optical coherence tomography (OCT) has become an important investigation tool for diagnosis and follow-up of retinal diseases and pathology of the anterior segment. The SL SCAN-1, Fourier domain OCT integrated into a slit lamp, enables to obtain OCT images simultaneous during a normal standard examination of the eye (anterior and posterior segment).

\section{What this study adds}

- The SL SCAN-1 is capable to scan through a handheld lens and through a three-mirror contact lens. With this, the peripheral retina and anterior chamber angle are within reach to obtain OCT images.

\section{Conflict of interest}

The authors declare no conflict of interest.

\section{Acknowledgements}

This work is published with the help of 'Edward en Marianne Blaauw Fonds'.

\section{References}

1 Stehouwer M, Verbraak FD, de Vries HR, Kok PH, van Leeuwen TG. Fourier domain optical coherence tomography integrated into a slit lamp; a novel technique combining anterior and posterior segment OCT. Eye (Lond) 2010; 24(6): 980-984.

2 Niyadurupola N, Broadway DC. Pigment dispersion syndrome and pigmentary glaucoma - a major review. Clin Exp Ophthalmol 2008; 36: 868-882.

3 Tektas OY, Lutjen-Drecoll E. Structural changes of the trabecular meshwork in different kinds of glaucoma. Exp Eye Res 2009; 88: 769-775.

4 Drexler W, Fujimoto JG. State-of-the-art retinal optical coherence tomography. Prog Retin Eye Res 2008; 27: 45-88.

5 Cense B, Nassif N, Chen T, Pierce M, Yun SH, Park B et al. Ultrahigh-resolution high-speed retinal imaging using spectral-domain optical coherence tomography. Opt Express 2004; 12: 2435-2447.

6 Jiao S, Knighton R, Huang X, Gregori G, Puliafito C. Simultaneous acquisition of sectional and fundus ophthalmic images with spectral-domain optical coherence tomography. Opt Express 2005; 13: 444-452.

7 Sarunic MV, Asrani S, Izatt JA. Imaging the ocular anterior segment with real-time, full-range Fourier-domain optical coherence tomography. Arch Ophthalmol 2008; 126: 537-542.

8 Vakhtin AB, Peterson KA, Kane DJ. Demonstration of complex-conjugate-resolved harmonic Fourier-domain optical coherence tomography imaging of biological samples. Appl Opt 2007; 46: 3870-3877. 
9 Dorairaj S, Liebmann JM, Ritch R. Quantitative evaluation of anterior segment parameters in the era of imaging. Trans Am Ophthalmol Soc 2007; 105: 99-108.

10 Leung CK, Li H, Weinreb RN, Liu J, Cheung CY, Lai RY et al. Anterior chamber angle measurement with anterior segment optical coherence tomography: a comparison between slit lamp OCT and Visante OCT. Invest Ophthalmol Vis Sci 2008; 49: 3469-3474.

11 Asrani S FAU, Sarunic MF, Santiago CF, Izatt J. Detailed visualization of the anterior segment using fourier-domain optical coherence tomography. Arch Ophthalmol 2008; 126(6): 765-771.
12 Fukuda S, Kawana K, Yasuno Y, Oshika T. Anterior ocular biometry using 3-dimensional optical coherence tomography. Ophthalmology 2009; 116: 882-889.

13 Gora M, Karnowski K, Szkulmowski M, Kaluzny BJ, Huber $\mathrm{R}$, Kowalczyk A et al. Ultra high-speed swept source OCT imaging of the anterior segment of human eye at $200 \mathrm{kHz}$ with adjustable imaging range. Opt Express 2009; 17: 14880-14894.

14 Wong HT, Lim MC, Sakata LM, Aung HT, Amerasinghe N, Friedman DS et al. High-definition optical coherence tomography imaging of the iridocorneal angle of the eye. Arch Ophthalmol 2009; 127: 256-260. 